\title{
MUJERES EN POLÍTICA: HACIA LA REIVINDICACIÓN DE LOS DERECHOS POLÍTICOS-ELECTORALES
}

\author{
Aldair Portillo ${ }^{1}$ \\ Jimena Bonilla Cabañas ${ }^{2}$
}

DOI: https://doi.org/10.5377//rd.v41i1.10505

\section{RESUMEN:}

La participación política de las mujeres se ha convertido en un fenómeno social y político a nivel internacional, puesto que siguen reclamando su lugar en la esfera pública y cargos de poder, en condiciones de igualdad y no discriminación. Ante este contexto, organismos internacionales y la mayoría de los Estados han accionado diligentemente en la búsqueda de mecanismos que permitan brindar una mejor protección y oportunidades a las mujeres en el mundo político, con el objetivo de lograr una aserción democrática y consolidar las bases del Estado de Derecho.

El presente artículo plasma un análisis sobre el contexto de la participación de la mujer en la política a nivel global y regional, los retos, desafíos y oportunidades en la búsqueda de la reivindicación de sus derechos políticos-electorales y el rol que juegan las cuotas de género para lograr una mayor representación en cargos de elección popular. De igual manera, se presentan propuestas en torno a la temática con el objetivo de lograr el fortalecimiento institucional de los partidos políticos, mayor participación de las mujeres en comicios electorales y cargos de poder público.

\section{PALABRAS CLAVE:}

Mujeres en Política, Cuotas de Género, Parlamentos, Derechos Políticos-Electorales.

Fecha de recepción: 30 de agosto de 2020 Fecha de aprobación:18 de noviembre de 2020

1 Estudiante de Derecho por egresar de la Universidad Nacional Autónoma de Honduras (UNAH).

Correo Electrónico: aldairportillo24@gmail.com

2 Doctora en Derecho Internacional, consultora y catedrática de la Universidad Nacional Autónoma de Honduras (UNAH).

Correo Electrónico: jimena.bonilla@unah.edu.hn 


\title{
WOMEN IN POLITICS: TOWARDS THE VINDICATION \\ OF POLITICAL-ELECTORAL RIGHTS
}

\section{Aldair Portillo ${ }^{3}$ Jimena Bonilla Cabañas ${ }^{4}$}

DOI: https://doi.org/10.5377//rd.v41i1.10505

\begin{abstract}
:
The political participation of women has become a social and political phenomenon at the international level, as they continue to claim their place in the public sphere and in positions of power, under conditions of equality and non-discrimination. In this context, international organizations and most of the States have acted diligently in the search for mechanisms that allow better protection and opportunities to be provided to women in the political world, with the objective of achieving democratic assertion and consolidating the bases of the State. of Law.

This article presents an analysis of the context of the participation of women in politics at the global and regional level, the challenges, challenges and opportunities in the search for the vindication of their political-electoral rights and the role played by the quotas of gender to achieve greater representation in popularly elected positions. Similarly, proposals are presented on the subject with the aim of achieving institutional strengthening of political parties, greater participation of women in electoral elections and positions of public power.
\end{abstract}

\section{KEYWORDS:}

Women in Politics, Gender Quotas, Parliaments, Political-Electoral Rights

Date received: August 30, 2020 Approval date: November 18, 2020

\footnotetext{
3 Law student to graduate from the Universidad Nacional Autónoma de Honduras (UNAH).

Email:aldairportillo24@gmail.com

4 Doctor in International Law, consultant and professor at the Universidad Nacional Autónoma de Honduras (UNAH).

Email: jimena.bonilla@,unah.edu.hn
} 


\section{INTRODUCCIÓN}

Los países a nivel global tienen una obligación y es la búsqueda del desarrollo de sus naciones, obligación que se materializa a través de la creación de organismos de integración y la adopción de normativa comunitaria a fin de garantizar un desarrollo más significativo e integral. Lo anterior, ha permitido crear vínculos económicos entre las diferentes regiones del mundo; el desarrollo de políticas conjuntas ha favorecido a los países en diferentes áreas, puesto que se ha generado mayor inversión y apertura de los mercados internacionales, fortaleciendo las exportaciones y logrando el desarrollo de la vida política y social de sus naciones.

En pleno siglo XXI los mayores desafíos se presentan en los temas de: ciencia y tecnología, arte, equidad de género y participación de la mujer en la política. Ante este contexto se deben tomar las acciones correspondientes en el marco de la consolidación democrática y de un desarrollo sostenible que permita crear sociedades más inclusivas y con mayores oportunidades; para ello, es necesario que quienes ostentan cargos de toma de decisiones integren en sus agendas públicas dichos temas y le otorguen la importancia que merecen.

Sin embargo, un tema que requiere una mayor atención a fin de contrarrestar los desafíos expuestos en el párrafo precedente es la participación de la mujer en la política, puesto que reviste la importancia necesaria y de mayor demanda en la palestra pública a nivel internacional, pues con el paso del tiempo la mujer ha sido pieza fundamental en la toma de decisiones y en la creación de políticas públicas para dar cumplimiento a las demandas de la población en nuestras sociedades.

Empero, la equidad de género y el papel de las mujeres en la política sigue siendo un tema sensible para nuestras poblaciones y para el mundo en su mayoría, debido a una cultura conservadora y a elementos históricos que siguen imperando en nuestras vidas de manera negativa y que impiden el progreso de nuestras naciones; a pesar de que las mujeres a lo largo de la historia han demostrado ser productivas, competentes y activas en diferentes esferas de participación.

Ante este escenario, el presente artículo plasma la actualidad, retos y oportunidades de la mujer en la política a nivel mundial y regional, los análisis correspondientes a las cutas de género, los mecanismos y recomendaciones en aras de convertirnos en una sociedad con mayores oportunidades en estadios de igualdad y no discriminación, comenzando su abordaje desde un marco conceptual que permita tener interpretaciones más amplias sobre el tema en cuestión.

De igual manera, se analiza lo referente a los derechos políticos-electorales y el número de mujeres en asientos parlamentarios, en el Poder Judicial y Poder Ejecutivo, tanto a nivel regional como local, para poder entender la dinámica de participación y los cambios que se están generando en este escenario en busca de la reivindicación de los derechos políticoselectorales de las mujeres.

Seguidamente se aborda lo referente al Corpus Iuris Internacional de los Derechos 
Políticos, a fin de conocer los instrumentos internacionalesque reconocen dichos derechos y que obligan a los Estados a establecer un marco de disposiciones legales para garantizar su pleno desarrollo; consecuentemente, se realiza un abordaje de la normativa interna de los países centroamericanos en lo relativo a las cuotas de género, para poder entender el número de mujeres en puestos de poder y si estas normativas ejercen cambios significativos o no.

Para concluir, la relevancia de este artículo radicará en el análisis estadístico de la participación de la mujer en política y, por consiguiente, en la presentación de propuestas para impulsar y convertir en una realidad la equidad de género y la autonomía de los derechos políticos de la mujer.

\section{MARCO CONCEPTUAL DE LA PARTICIPACIÓN POLÍTICA DE LAS MUJERES.}

El progreso y consolidación de una democracia depende del grado de integración y complementariedad en la toma de decisiones de sus liderazgos y en la efectiva igualdad entre hombres y mujeres. De lo anterior debe entenderse que los países deben aprovechar su diversidad para lograr un crecimiento gradual y efectivo partiendo de la igualdad de género como un pilar fundamental en el fortalecimiento democrático de nuestras naciones.

Es por ello por lo que el paso del tiempo y los desafíos que presenta el mundo actual demandan cada día mayor participación de la mujer en las diferentes esferas de desarrollo de la vida humana, siendo la política una necesidad imperante en nuestro entorno. Sin embargo, la participación de la mujer se ha visto limitada históricamente y a pesar de los esfuerzos políticos por lograr brindar mayores oportunidades, el progreso ha sido minoritario, existiendo sociedades a nivel mundial donde a la mujer aún no se le reconoce el derecho al voto.

Debemos ser conscientes que la problemática entorno a las limitaciones que tienen las mujeres en la política, no radica en el hecho de que la mujer ha dejado su casa y ha dejado de procrear hijos para involucrarse en espacios tradicionalmente ocupados por hombres. El meollo del asunto radia en el abordaje conceptual de su actividad, pues la mujer ha sido víctima de ataques constantes a su dignidad, los cuales obstaculizan el logro de sus propósitos.

Ante un contexto de desigualdad por su condición de género, la lucha por ejercer su derecho de voto y decisión comienza a tomar fuerza con la publicación de la Declaración de los Derechos de la Mujer y de la Ciudadana (1791). Aquí adquiere relevancia la conquista de las mujeres a ejercer el sufragio, puesto que este precedente aceleró la actuación de la mujer en el ámbito político y en los estadios de decisiones. El hecho más significativo y que marcó un precedente a nivel mundial lo encontramos con la llegada al poder de Indira Gandhi a la jefatura de gobierno de su país, India.

La participación de la mujer va más allá de ejercer el sufragio en los diferentes procesos electorales, como derecho político; 
su participación radica en el reconocimiento de sus capacidades, en la materialización de sus ideas y en la igualdad en torno al sexo masculino en la política nacional. Comprende, además, el empoderamiento de la mujer y la representación efectiva de su género en las democracias nacionales y en la búsqueda de la justicia, el progreso y la paz de nuestros países.

En este sentido, es necesario que se logre erradicar los estereotipos sobre la mujer, los diferentes modos en que se construyen las relaciones de poder e identidad de los sujetos en los espacios de la vida política y el cambio de imagen en la receptividad de la sociedad en general; es decir, que las mujeres puedan hacer uso pleno del ejercicio de la ciudadanía en condiciones de igualdad y equidad.

De acuerdo con el informe realizado por el Instituto holandés para la democracia multipartidaria (NIMD por sus siglas en inglés) y el Programa de las Naciones Unidas para el Desarrollo (PNUD por sus siglas en inglés) sobre: Mujeres y política, claves para su participación y representación (2018), "la participación política de las mujeres es un derecho fundamental, y asegurar su ejercicio es una obligación del Estado". De lo anterior debe entenderse que no existe argumento jurídico que limite la participación de las mujeres en la política nacional y que son los Estados los obligados a crear las condiciones de participación y las instituciones especiales que garanticen el ejercicio de estos en condiciones de igualdad. Para ello, se deben crear disposiciones o normativas orientadoras para los partidos políticos para que se construya de manera progresiva una nueva cultura política donde se respete y garantice el papel de las mujeres en este ámbito.

Las mujeres han demostrado que son capaces de liderar cualquier escenario de la vida política social y cultural de un país y que a través de sus experiencias, conocimientos $\mathrm{y}$ visiones pueden conducir al progreso y a la mejora de las condiciones de vida de sus ciudadanos. Además, son fundamentales en la vida económica de nuestros países, ya que cada día son más las mujeres emprendedoras y empresarias que dinamizan el flujo de activos financieros.

Aunado a lo anterior, el Consejo de Seguridad de las Naciones Unidas reconoció el importante papel que juegan las mujeres en la construcción de la paz y en la resolución de conflictos armados alrededor del mundo, pues estas aportan ideas frescas y viables, permitiendo procesos de diálogo y negociación entre los diferentes actores en conflicto, a fin de poder lograr espacios de comunicación más asertivos que conduzcan a lograr mayor seguridad nacional, respeto a los derechos humanos y la tranquilidad de las personas en sus territorios.

Empero, en la actualidad las mujeres siguen siendo voces subrepresentadas, pues en la mayoría de los casos las esferas de participación son limitadas y si las hay, tienen que vencer una serie de obstáculos para poder optar y llegar a un cargo de elección popular o a un puesto ejecutivo dentro de la administración pública.

Es por ello por lo que se han incorporado las cuotas de género, las cuales son una 
forma de acción positiva cuyo objetivo es garantizar la efectiva integración de mujeres en cargos electivos de decisión de los partidos políticos y del Estado. Es una medida de carácter compulsivo, que obliga a incorporar mujeres en listas de candidaturas o en listas de resultados electorales, y transitorio, puesto que supone una vigencia sujeta a la superación de los obstáculos que impiden una adecuada representación de mujeres en los espacios de poder y representación política. (Fernández, 2011)

La Dra. Anna María Fernández (2011) establece la existencia de tres tipos de cuotas para lograr la paridad política y lograr la reivindicación de los derechos políticos de las mujeres, a saber: a) Constitucionales: consagradas en leyes fundamentales $y$ constituciones; b) Legislativas: establecidas en leyes y en leyes electorales; c) Partidos políticos: para la selección cargos internos. Pero, para lograr resultados concretos al respecto, es necesario que pueda existir una normativa vigente y el ente encargado de dar cumplimiento a la misma, aquí es vital la participación de los órganos electorales.

\section{EN LA BÚSQUEDA DE LA AUTONOMÍA POLÍTICA}

A lo largo del tiempo, las mujeres siempre se han mantenido activas en la vida política de sus naciones, unas más que otras y en diferentes escenarios; actualmente, el problema radica en la autonomía del ejercicio de sus derechos políticos, premisa que se materializa con la poca representación de estas en la esfera pública. Según datos de las Naciones Unidas (ONU Mujeres) para enero de 2020 el
24.9\% de los escaños parlamentarios (entre la Cámara única y el Senado) son ocupados por mujeres y solo 57 de los 278 Parlamentos son encabezados por las mismas; $y$, a nivel ejecutivo, solo 12 mujeres ocupan el cargo de Jefas de Gobierno y 10 el de Jefas de Estado.

Según datos del Inter-Parliamentary Union (IPU 2020) solo cuatro países cuentan con más del $50 \%$ de mujeres parlamentarias: Ruanda con $61.3 \%$, Cuba con el 53.2\%, Bolivia con un $53.1 \% \mathrm{y}$, Emiratos Árabes Unidos con el $50 \%$. Los datos anteriores denotan que sigue existiendo la subrepresentación en los cargos de elección popular, situación que se agrava más, si consideramos el número de países y asientos parlamentarios a nivel global.

Desde una óptica regional, los países nórdicos han liderado la participación política de las mujeres en cargos de elección popular, a la fecha cuentan con porcentaje de participación del $43.9 \%$, pero, si se analiza al continente europeo en su conjunto, la cifra desciende al $30.1 \%$ en la Cámara única o baja, al 29.1\% en la Cámara alta o el Senado y al $29.9 \%$ si se combinan ambas Cámaras. Sin embargo, dichos porcentajes han sido superados por América, que para 2020 cuenta con un $31.1 \%$ de mujeres parlamentarias en las Cámaras únicas, con un $32.1 \%$ en el Senado y con un $31.3 \%$ en las Cámaras combinadas. Actualmente, es la región del mundo con mayor avance en la búsqueda de la igualdad y paridad política; el crecimiento se debe a la mayor participación de las mujeres en la vida económica, en un mayor grado de escolaridad y en la incorporación de las políticas de género en la normativa electoral adoptadas por algunos países latinoamericanos. Empero, 
la mayor desigualdad en el ejercicio de los derechos políticos electorales por parte de las mujeres se da en Asia, Medio Oriente, Norte de África y el Pacifico, donde las cifras no superan el $20 \%$ de mujeres en asientos parlamentarios (ONU Mujeres, 2020).

Por otro lado, según la Comisión Económica para América Latina (CEPAL, 2019), Latinoamérica en 2012 contaba con un $25 \%$ de mujeres electas a nivel de concejales, con $24.3 \%$ de representatividad en el Máximo Tribunal de Justicia y con el $11.7 \%$ a nivel de alcaldesas; para el 2014, cinco países eran gobernados por mujeres y para 2020 la cifra se reduce a tres mandatarias; los datos anteriores son preocupantes porque existe una regresividad en la búsqueda de la igualdad política de las mujeres en la región. La poca representatividad de las mujeres en los diferentes Poderes del Estado se debe en su mayoría, a la falta de: aceptación sociocultural de las mujeres elegidas, confianza $y$, discriminación de iure y de facto por órganos estatales y servidores públicos.

Incluso cuando se ha demostrado la existencia de productividad, participación y competencias, se les concibe como incompetentes y se les busca imponer calificativos peyorativos, lo que deriva conductas violentas de invisibilización, obstaculización y supresión; razones por las que, en el año 2018, solo dos mujeres fueron electas a nivel presidencial: Paula Mae Weekes en marzo de 2018 toma posesión de la Presidencia de Trinidad y Tobago y en mayo del mismo año, Mia Amor Mottley asume el cargo de Primera Ministra de Barbados, ambas se convierten en la primera mujer en ocupar dicho cargo en sus respectivos países. En noviembre de 2019 Jeanine Áñez Chávez, asume la Presidencia por sucesión constitucional de Bolivia y se convierte en la segunda mujer en ocupar dicho cargo. Lo que conlleva a un retroceso en el empoderamiento y participación de las mujeres en la Administración Pública en comparación con el año 2014.

Los expertos advierten que, las mujeres mandatarias enfrentan retos mayores en sus periodos de gobierno en la figura presidencial, como vicepresidencial, teniendo limitaciones de distinto tipo, tanto en su administración como en los aspectos culturales entre los que resalta el machismo y el sexismo, los cuales son difíciles de modificar. Ante este contexto, es de carácter imperativo la creación de marcos normativos, que se pongan en acción planes estratégicos y se implementen controles de ejecución para contrarrestar la problemática. Para ello, es necesario intensificar esfuerzos que puedan ser analizados y compartir las experiencias locales y estrategias que permitan identificar la violencia en la política y las oportunidades que se tienen.

En 1979 la Organización de las Naciones UnidascrealaConvención sobrelaEliminación de todas las formas de Discriminación contra la Mujer (CEDAW). Y, en 1994 se adopta la Convención Interamericana para Prevenir, Sancionar y Erradicar la Violencia contra la Mujer, conocida como Convención de Belém do Pará. Al respecto, investigaciones del InterParliamentary Union (IPU), muestra que las mujeres obtuvieron el $30 \%$ de las bancadas en los 20 países donde se aplicaron cuotas en el 2017; mientras que solo el $15.4 \%$ de 
los escaños fueron ganados por mujeres en los 16 países donde no se fijaron objetivos. Para el 2020, la mayoría países de América Latina y el Caribe tienen leyes de cuotas para mejorar la participación de las mujeres en cargo de elección popular; a pesar de ser la primera región del mundo con mayor número de parlamentarias, la voluntad política por lograr la igualdad y autonomía de las mujeres en esta esfera varía por país.

En Centroamérica una serie de factores culturales e históricos han impedido que en la región se trate el tema como una prioridad. En esta línea argumentativa, el reconocimiento de la ciudadanía de la mujer centroamericana comienza con el derecho al voto, siendo El Salvador quien marcó la pauta reconociéndolo en 1939, seguido por República Dominicana en 1942; Costa Rica en 1944; Panamá en 1946 de manera plena; Nicaragua en 1995 y Honduras en 1957; Guatemala lo hizo hasta en 1965, sin restricciones. La conquista del derecho al sufragio marca un punto de inflexión entre una sociedad indiferente y la búsqueda de la igual política.

Es de suma importancia resaltar la diferencia entre la cuota de participación, ya sea legislada o voluntaria, y; la representación efectiva de las mujeres en los cargos de elección popular y titulares de cualquier poder estatal. La primera se refiere al porcentaje de participación de las mujeres en la política, fijada por la ley o por los partidos políticos; mientras que la segunda depende de circunstancias modificantes en su entorno particular (como: la cultura, elementos históricos, sanción y alternancia y en relación con los procesos electorales (Archenti, 2011).
Según datos de la CEPAL, para 2020, a nivel del Poder Ejecutivo, dos países avanzan de manera significativa en la búsqueda del equilibrio político, Nicaragua con un $56.3 \%$ y Costa Rica con el 55.2\%, ambos con una cuota legislada del 50\%. Mientras que Honduras y El Salvador se posicionan con el $26.3 \%$, Panamá, con $20 \%$; siendo Guatemala y Belice los más bajos con el $15.6 \%$ y $14.3 \%$ respectivamente, cifras que distan mucho del equilibrio en la paridad y alternancia política, a pesar de contar con leyes de cuota.

En datos del Instituto para la Democracia y Asistencia Electoral (IDEA, 2020), a nivel legislativo, encontramos variaciones en el número de asientos parlamentarios ocupados por mujeres, Nicaragua lidera la lista con el $47 \%$ (2016), seguido por Costa Rica con el 46\% (2018), se suma El Salvador con el 33\% (2018). Panamá se posiciona en el cuarto lugar con un 23\% (2019) y Honduras con el $21 \%$ (2017); cifras desoladoras en la búsqueda de la representación igualitaria y autonomía política de la mujer en la región. Pero, la mayor preocupación recae en Guatemala con un 19\% (2019) y en Belice, quien cuenta con apenas 9.4\% (2015). Entre los factores que influyen en este contexto, nos encontramos con la violencia hacia la mujer, el machismo y el grado de educación de los ciudadanos, en especial los del área rural que no se adaptan al cambio de papeles en la sociedad.

En cuanto al Poder Judicial, las cifras toman rumbos diferentes en este escenario, Guatemala se convierte en el primer país con mayor número de mujeres en la Institución de mayor Jerarquía Judicial, como lo es la Corte Suprema de Justicia, con el 41.7.8\%; mientras 
que la media se mantiene en $33.3 \%$ para $\mathrm{El}$ Salvador, Honduras y Costa Rica; Nicaragua se posiciona con el 31.3\%; seguido de Belice con el $16.7 \%$ y de último se posiciona Panamá con el $11.1 \%$

En definitiva, la Ley de Cuotas en los países centroamericanos ha contribuido a que las mujeres tengan igual representación en los Poderes del Estado, en igualdad de condiciones y oportunidades, en aras de lograr la reivindicación de los derechos políticos y la autonomía de las mujeres en la política. Países como Nicaragua y Costa Rica, actualmente son modelos en los procesos de la paridad política a nivel centroamericano, latinoamericano y mundial. Lo anterior, se debe a la materialización efectiva de la normativa jurídica implementada en sus ordenamientos jurídicos internos; constituyendo a la vez, un gran paso en los procesos de integración centroamericana. Mientras que en países como, Panamá, Belice y República Dominicana siguen teniendo desafíos en este tema.

\section{CORPUS IURIS DE LA} PARTICIPACIÓN POLÍTICA DE LAS
MUJERES Y LA CUOTA DE GÉNERO.

Con el paso del tiempo, los organismos internacionales han coadyuvado a que los Estados adopten disposiciones legales dentro de sus ordenamientos jurídicos internos que permitan una tutela efectiva de los derechos políticos de las mujeres y a la vez, que se sigan creando los mecanismos especializados para materializar dichas disposiciones. Lo anterior, con el fin de orientar, fijar lineamientos bases y promover los espacios de participación ciudadana en la vida democrática de nuestros países.

Por tal razón, para poder garantizar la participación política de las mujeres y hacer prevalecer la cuota de género, se ha creado un Corpus Iuris de los Derechos Políticos que contempla un marco jurídico de carácter internacional y consecuentemente, una base normativa de carácter nacional; donde se fijan disposiciones legales que permiten establecer una serie de derechos políticos de carácter igualitario entre hombres y mujeres y obligaciones estatales para dar cumplimiento a los mismos.

En las siguientes líneas, se analizan los instrumentos internacionales que plasman desde un enfoque de los derechos humanos, el derecho de la participación política de los ciudadanos sin distinción alguna y en igualdad de condiciones, dichos tratados vienen a fortalecer la normativa nacional sobre el tema en cuestión, la cual será analizada en un segundo momento.

Para entrar en materia, el Pacto Internacional de los Derechos Civiles y Políticos (instrumento de las Organización de las Naciones Unidas) recoge en su artículo 25 la protección de los derechos políticoselectorales, mismos que han sido adoptados de manera literal en el Sistema Interamericano, siendo estos reconocidos en la Convención Americana de Derechos Humanos (CADH), en la cual se interpreta de manera más amplia la cláusula democrática contenida en la Carta de la Organización de Estados Americanos (OEA) con respecto a estos derechos. Sin menoscabo de lo establecido 
en la Convención Americana de los Derechos del Hombre (DADDH), a fin de poder lograr la consolidación de la democracia formal y representativa en el continente americano.

En este sentido, en su artículo 23, la CADH establece el derecho a participar en la dirección de asuntos públicos, de votar y ser electo en el marco del principio de igualdad $\mathrm{y}$ no discriminación $\mathrm{y}$, a tener acceso en condiciones de igualdad a las funciones públicas de su país; lo anterior, a la luz de las obligaciones contenidas en el artículo 1.1. y 2 convencional, es decir, la obligación de respeto y garantía por un lado y la de adoptar disposiciones internas y crear las instituciones especializadas, por el otro.

En el mismo orden de ideas, la Convención sobre la Eliminación de Todas las Formas de Discriminación Contra la Mujer (CEDAW, por sus siglas en ingles), plasma en su artículo 4 las medidas especiales que deben tomar los Estados encaminadas a garantizar la igual de facto entre hombres y mujeres; complementariamente, en el artículo 7 del mismo instrumento, se establece la obligación de los Estados de adoptar todas las medidas necesarias a fin de eliminar todo tipo de discriminación contra la mujer en la vida política y pública del país $\mathrm{y}$, de garantizar los derechos políticos citados en los párrafos precedentes.

En igual forma, la Convención Interamericana para Prevenir, Sancionar y Erradicar la Violencia contra la Mujer (Belém do Pará) establece en su artículo 4 que las mujeres tienen el derecho al reconocimiento, goce, ejercicio y protección de todos los derechos humanos y a las libertades consagradas por los instrumentos regionales e internacionales sobre derechos humanos. Incluyendo, el derecho a tener igualdad de acceso a las funciones públicas de su país, a participar en los asuntos públicos y a la toma de decisiones.

Por otro lado, la Resolución 1325 del Consejo de Seguridad de las Naciones Unidas (2000), exhorta a los Estados a permitir una mayor participación de las mujeres en todos los niveles, puesto que, estas juegan un papel trascendental en la prevención y resolución de conflictos, y, por tanto, es importante que participen por igual en los procesos de construcción de la paz y la seguridad nacional.

En Centroamérica la participación política electoral de las mujeres es un tema que en los últimos años se ha incorporado a la agenda pública de los países del istmo y del Sistema de Integración Centroamericana (SICA). Si bien es cierto ha habido avances significativos en lo relativo a la cuota de poder, también no es menos cierto que el compromiso de los países en la mayoría de los casos es de carácter formalista, pues no se reflejan cambios significativos en las leyes electorales internas y de los partidos políticos, que permitan mayores espacios de participación para las mujeres en condiciones de igualdad y no discriminación.

Es por ello, que para garantizar la participación política de las mujeres de manera efectiva se han creado las denominadas "cuotas de género" que no es más que el porcentaje obligatorio en los puestos de elección popular que deben cumplir los partidos políticos para 
poder participar en los comicios electorales, misma que se ha institucionalizado de manera parcial y progresiva en los países de la región.

Guatemala y Belice son los países centroamericanos que en pleno siglo XXI no cuentan con una cuota de género en su ley electoral, lo único que garantiza su Constitución Política es el derecho al voto para las mujeres. Mientras que El Salvador establece una cuota constitucional del 30\% para los comicios electorales de la Asamblea Legislativa, empero, dicha disposición no especifica los lugares de la papeleta electoral, por lo tanto, están expuestas a que sean relegadas a los últimos lugares y su participación sea de carácter simbólico.

Por otra parte, en Honduras la lista de candidatos de los partidos políticos para las elecciones del Poder Legislativo debe incluir una cuota de por lo menos $40 \%$ de mujeres candidatas, de infringir el partido con dicha disposición se le aplicará una multa del 5\% del financiamiento estatal para todos los partidos.

Nicaragua y Costa Rica constituyen el mayor ejemplo de respeto por los derechos políticos electorales en la región y por la consolidación de una cultura democrática basada en los principios de igualdad y no discriminación entre hombres y mujeres, por lo tanto, la cuota de género es del $50 \%$, cuota que viene a fortalecer los principios de inclusión, participación y paridad en dichos países y garantizar la alternancia y representatividad.

Finalmente, Panamá establece en su ley electoral una cuota del 50\% que debe ser cumplida por todos los partidos políticos; sin embargo, no se establece un sistema que permita la alternancia y la igualdad de derechos al momento de integrar una planilla electoral

En suma, es importante que se siga aspirando a la igualdad de derechos y obligaciones políticos-electorales en nuestra región. Para romper la brecha de desigualdad entre hombres y mujeres, los ciudadanos debemos hacer eco de la normativa nacional e internacional en esta materia a fin de hacer prevalecer los derechos reconocidos y su correcta aplicación por parte de las autoridades nacionales de cada uno de los países del istmo.

\section{ALGUNAS PROPUESTAS PARA FORTALECER LA PARTICIPACIÓN POLÍTICA DE LAS MUJERES}

Para poder fortalecer la participación de las mujeres y lograr la reivindicación de sus derechos políticos-electorales, es necesario que se puedan articular esfuerzos con los diferentes sectores de la sociedad, para romper con esas barreras que limitan su participación y poca representación en asientos parlamentarios y demás cargos de la administración pública, a fin de que se puedan establecer programas integrales que permitan la creación de oportunidades para todas en igualdad de condiciones. Así, por ejemplo, se pueden implementar las siguientes:

\section{Programas de formación especializados:} enfocados en el empoderamiento de niñas y jóvenes en la política, en su formación y en la necesidad de brindar los mecanismos que les permitan desarrollar sus capacidades y 
aptitudes para preparar su camino a la palestra pública. Estos deben ser desarrollado por los partidos y movimientos políticos y se deben crear indicadores para poder evaluar su avance y oportunidades de mejora.

Escuela de Mujeres Parlamentarias: si bien es cierto en algunos países de la región ya se implementó, es necesario su fortalecimiento en todos los países del continente, formación que debe ser de manera integral y como un requisito previo a poder optar a un asiento parlamentario; una vez formando parte del Poder Legislativo, se debe continuar con dicho proceso de formación e ir mejorando la dinámica de aprendizaje; lo anterior con el objetivo de crear mujeres más empoderadas, con mayores competencias y conscientes de su papel en la sociedad.

\section{Congresos de Liderazgo Político:} los países de la región deben apostar al intercambio de ideas y al fortalecimiento de lazos de hermandad entre las mujeres del continente americano. Se deben aprovechar las experiencias individuales por país y enriquecerse de las diferencias para lograr converger hacia un proceso de inclusión que conlleve a la creación de redes de mujeres en cargos de poder y que estas coadyuven al fortalecimiento de una cultura política más democrática y sin discriminación alguna.

Clausulas informativas: los órganos electorales deben jugar un papel protagónico en la carrera por lograr la paridad de género en nuestras naciones, para ello, es necesario que puedan adoptar todas las medidas de carácter legal y utilizar todos los medios a su alcance que permitan formar, informar y educar a la población en materia política y sobre todo en la necesidad de lograr la igualdad de participación entre hombres y mujeres en todos los espacios de participación político-electoral; por tal motivo, las cláusulas informativas en redes sociales, medios de comunicación oral y escrita constituyen una excelente alternativa para lograr el cumplimiento de dichos objetivos.

\section{LA IMPORTANCIA DE LAS CUOTAS DE GÉNERO REGLAMENTADAS}

Las cuotas de género juegan un papel fundamental para lograr mayor representación de mujeres en cargos de elección popular y puestos ejecutivos dentro de la administración pública. Si bien es cierto, existe un auge significativo en cuanto a la participación política de las mujeres en la región, no es menos cierto, que dista mucho para lograr una autonomía política efectiva y permanente. Empero, la normativa vigente, como la Ley de Cuotas, ha sido uno de los mecanismos más efectivos en la búsqueda de la paridad de género, en este contexto, debe existir un sistema de monitoreo para el cumplimiento de las acciones enmarcadas en dicho marco normativo.

Consecuentemente, no basta que los Estados adopten en sus jurisdicciones internas la Ley de Cuotas, sino que es imperativo que estas puedan ser reglamentadas, de aplicación inmediata y obligatoria en todos los escenarios de participación. Esta regulación debe contener entre otras cosas, el porcentaje de mujeres en cargos de elección, la alternabilidad en papeletas electorales y la creación de un órgano especializado que dé 
cumplimiento a dichas disposiciones legales, para garantizar con ello un pleno goce de los derechos políticos-electorales en igual de condiciones y no discriminación en la vida política de nuestros países.

De igual forma, se deben establecer obligaciones generales para las autoridades electorales, para los partidos políticos y para las organizaciones de sociedad civil; a fin de brindar una tutela efectiva en el marco del respeto y garantía de los derechos mencionados en el párrafo precedente, para que mediante la articulación de esfuerzos y acciones complementarias que de paso a una nueva cultura política. Empero, no se debe dejar por fuera el tema de las sanciones por el incumplimiento de la normativa vigente en materia política-electoral, pues resulta de suma importancia la adopción de un mecanismo de control y supervición a fin de lograr el cumplimiento material de la Ley de Cuotas y su Reglamento y no establecerlo por pura formalidad y con un carácter ilusorio.

En el mismo orden de ideas los partidos políticos e iniciativas de sociedad civil en materia de participación ciudadana juegan un papel imprescindible y principalmente quienes ostentan el liderazgo de los mismos, puesto que deben realizar una serie de acciones encaminadas a poder integrar la paridad de género en sus programas formativos y planes operativos en general, en tres momentos claves, a saber: a) previo a las elecciones o procesos de selección de candidatos, b) durante los procesos electorales y, c) post elecciones. Según el NIMD y el PNUD (2018), los partidos y movimientos políticos deben "avanzar en la aplicación progresiva de los principios constitucionales de paridad, alternancia y universalidad en todas las listas a corporaciones de elección popular y circunscripciones, incluso si su aplicación no ha sido reglamentada mediante una ley”.

Ahora bien, la equidad de género debe ser un eje transversal en los sistemas educativos para acabar con los problemas de violencia y discriminación de la mujer en la política; lo anterior, implica un mayor grado de voluntad política y la adopción de políticas públicas de carácter integral. En el mismo sentido, se deben crear las instituciones necesarias que permitan mayores oportunidades a las mujeres y el acceso a la justicia sin distinción alguna.

La autonomía política de las mujeres implica el reconocimiento de su ciudadanía. Si nuestros países avanzan en este tópico, por ende, avanzarán en su proceso de integración nacional y regional. Las mujeres agregan equilibrio en la toma de decisiones y permiten el uso de mejores prácticas políticas que conllevan a la consolidación de la democracia y a la creación de mejores oportunidades para todos, especialmente para aquellos que no tienen voz.

En síntesis, tenemos un enorme reto para vencer la brecha de desigualdad política que existe en el presente, para lograr mayor representación en cargos de elección popular, mayor participación política y mayor número de mujeres en cargos ejecutivos. Como ciudadanos tenemos un deber ineludible de contribuir a la generación de espacios más receptivos en nuestros escenarios políticos, comicios electorales en igualdad de condiciones y democracias más inclusivas. 


\section{BIBLIOGRAFÍA}

Vélez, Anarella. (2019) Las mujeres y la insurrección en el siglo XXI. Estudios de la Mujer. Recuperado de: https:// estudiosdelamujer.wordpress.com/.

ONU MUJERES. Conferencias mundiales sobre la Mujer. Recuperado de: http:// www.unwomen.org/es/how-we-work/ intergovernmental-support/worldconferences-on-women

Becerra, Malacalza, Mendoza y Soria. (2007) Participación política de las mujeres y rol de las ONG. Asociación Latinoamericana de Organizaciones de Promoción del Desarrollo. Recuperado de: http://www. gloobal.net/iepala/gloobal/fichas/ficha.php $\underline{? \mathrm{id}=3549 \& \text { entidad }=\text { Textos } \& \text { html }=1}$

Fernández, A. M. (2011). Las cuotas de género y la representación política femenina en México y América Latina. Impactos (México, D.F). Documento disponible en: http://www.scielo.org.mx/pdf/argu/ v24n66/v24n66a10.pdf

IDEA (2020) Cuotas de género. Archivo disponible en: https://www.idea.int/datatools/data/gender-quotas/database.

NIMD \& PNUD (2018) Mujeres en política: claves parasuparticipacióny representación. Archivo disponible en: https://www. refworld.org.es/pdfid/5c3f6d524.pdf

Lissardy, Gerardo. (2017) América Latina se queda sin mujeres presidentas: ¿cuánto cambió realmente la región en machismo, sexismo y poder? Recuperado de: https:// www.bbc.com/mundo/noticias-americalatina-42406642

ONU MUJERES (2019). Women rising: Women's activism that has shaped the world as you know it. Recuperado de: https:// medium.com/we-the-peoples/womenrising-womens-activism-that-has-shapedthe-world-as-you-know-it-5850cd112bac

La Nación. (2014) El rol de la mujer en la Política. Recuperado de: https:// www.nacion.com/opinion/foros/ el-rol-de-la-mujer-en-la-politica/ YODS3PXVTFGSTBHAF7EN7QAQSE/ story/

ONU MUJERES (2019) Hechos y cifras: liderazgo y participación política: Presencia de mujeres en los Parlamentos. Recuperado de: http://www.unwomen.org/es/what-wedo/leadership-and-political-participation/ facts-and-figures

CEPAL. Violencia hacia las mujeres en la política: Herramientas para su prevención y abordaje. Recuperado de: https://www. cepal.org/es/datos-y-estadisticas

CEPAL. Observatorio de América Latina y el Caribe. Políticas de Igualdad de género. Recuperado de: https://oig.cepal.org/es/ politicas-justas

IPU. (2020) Women in national parliaments. Recuperado de: http://archive.ipu.org/ wmn-e/classif.htm 
Archenti, N. (2011). La paridad política en América Latina y el Caribe: Percepciones y opiniones de los líderes de la región. Naciones Unidas (2011). Recuperado de: https://repositorio.cepal.org/bitstream/ handle/11362/5835/S1100674 es.pdf

CEPAL. Observatorio de Igualdad de Género de América Latina y el Caribe. (2020) Indicadores: Poder ejecutivo: porcentaje de mujeres en los gabinetes ministeriales. Recuperado de: https://oig.cepal.org/es/ indicadores/poder-ejecutivo-porcentajemujeres-gabinetes-ministeriales

CEPAL. Observatorio de Igualdad de Género de América Latina y el Caribe. (2020) Indicadores: Poder judicial: porcentaje de mujeres ministras en el máximo Tribunal de Justicia o Corte Suprema. Recuperado de: https://oig.cepal.org/es/indicadores/poderjudicial-porcentaje-mujeres-ministrasmaximo-tribunal-justicia-o-corte-suprema

Pinget, Yasmin \& Garrido, Carmen. (2019) Mujeres parlamentarias en América Latina y el Caribe: Exigencias y Equilibrios. Condistintosacentos. Recuperado de: http://www.condistintosacentos.com/ mujeres-parlamentarias-en-america-latinay-el-caribe-exigencias-y-equilibrios/\# ftn1 